\title{
Ordenanza municipal sobre las normativas ambientales para regular la contaminación hídrica
}

\section{Municipal ordinance on environmental regulations to regulate water pollution}

\author{
Alexandra Dolores Molina-Manzo \\ ub.alexandramolina@uniandes.edu.ec \\ Universidad Regional Autónoma de los Andes, Babahoyo \\ Ecuador \\ https://orcid.org/0000-0001-5015-9739 \\ Génesis Gaibor-Verdezoto \\ genesisgaibor1997@gmail.com \\ Universidad Regional Autónoma de los Andes, Babahoyo \\ Ecuador \\ Diana Rosero-Mora \\ roseromoradiana@gmail.com \\ Universidad Regional Autónoma de Los Andes, Babahoyo \\ Ecuador
}

Recibido: 31 de octubre de 2020

Revisado: 10 de octubre de 2020

Aprobado: 05 de diciembre de 2020

Publicado: 10 de diciembre de 2020 


\title{
RESUMEN
}

El objetivo de la presente investigación fue fundamentar jurídica y teóricamente, que la contaminación ambiental y la falta de ordenanzas municipales es una causa de vulneración a los derechos de la naturaleza. El mismo se desarrolló en el Cantón Montalvo, con ubicación en la Provincia de los Ríos, donde existe alta contaminación hídrica que vulnera los derechos del medio ambiente y de la población. Desde el paradigma cuantitativa y la perspectiva del método analítico-sintético, lo que permito trabajar a partir de un objeto de estudio (normativa ambiental), la descomposición analítica de sus partes para luego ser interpretadas. Se procedió metodológicamente desde un tipo descriptivo documental con diseño bibliográfico. Se concluyó que el Gobierno Autónomo Descentralizado Municipal del Cantón Montalvo no cuenta con ordenanzas que ayuden a disminuir, proteger y sancionar a los contaminantes de ríos, esteros y quebradas y peor aún con trabajos que garanticen un medio ambiente adecuado para los pobladores del Cantón.

Descriptores: Contaminación del agua, higiene ambiental, régimen jurídico. (Palabras tomadas del Tesauro UNESCO).

\begin{abstract}
The objective of the present investigation was to establish, legally and theoretically, that environmental contamination and the lack of municipal ordinances is a cause of violation of the rights of nature. It was developed in the Cantón Montalvo, located in the Province of Los Ríos, where there is high water pollution that violates the rights of the environment and the population. From the quantitative paradigm and the perspective of the analytic-synthetic method, which I allow to work from an object of study (environmental regulations), the analytical decomposition of its parts and then be interpreted. We proceeded methodologically from a descriptive documentary type with bibliographic design. It was concluded that the Autonomous Decentralized Municipal Government of the Canton Montalvo does not have ordinances that help reduce, protect and sanction the pollutants of rivers, streams and streams and worse still with works that guarantee an adequate environment for the inhabitants of the Canton.
\end{abstract}

Descriptors: Water pollution, environmental hygiene, legal regime. (Words taken from the UNESCO Thesaurus). 


\section{INTRODUCCIÓN}

El Cantón Montalvo, con ubicación en la Provincia de los Ríos, donde existe alta contaminación hídrica que vulnera los derechos del medio ambiente y de la población de la ciudad y sus alrededores. Es importante la colaboración de las autoridades competentes para la ejecución de proyectos ya que con su ayuda se podrán aplicar de manera inmediata las normas destinadas a la protección y disminución de la contaminación ambiental que se ha producido en los últimos años. Se consagra a la naturaleza como sujeto de derecho, tal como lo estipula el Artículo 10.- de la Constitución: Las personas, comunidades, pueblos, nacionalidades y colectivos son titulares y gozarán de los derechos garantizados en la Constitución y en los instrumentos internacionales.

La naturaleza será sujeto de aquellos derechos que le reconozca la Constitución. De igual forma en su Capitulo séptimo artículo 71: La naturaleza o Pacha Mama, donde se reproduce y realiza la vida, tiene derecho a que se respete integralmente su existencia y el mantenimiento y regeneración de sus ciclos vitales, estructura, funciones y procesos evolutivos.

Toda persona, comunidad, pueblo o nacionalidad podrá exigir a la autoridad pública el cumplimiento de los derechos de la naturaleza. Para aplicar e interpretar estos derechos se observarán los principios establecidos en la Constitución, en lo que proceda.

El Estado incentivará a las personas naturales y jurídicas, y a los colectivos, para que protejan la naturaleza, y promoverá el respeto a todos los elementos que forman un ecosistema. (Constitución de la República del Ecuador,2008).

La crisis ecológica no solo afecta a una parte de los territorios, sino que es un problema que se ha creado a nivel mundial, por tal situación es necesario tomar conciencia del daño causado a la naturaleza, por lo que es preciso analizar y plantear propuestas que sean coherentes con el hombre y su entorno. Por ello, las limitaciones de disponibilidad de agua en términos de calidad y el importante crecimiento demográfico nacional están ejerciendo una fuerte presión sobre las zonas altas de páramos de donde se originan las fuentes de agua primarias, generando como 
consecuencia la sobre explotación del recurso y el deterioro de su cobertura vegetal natural en las áreas de recarga. (Terneus-Jácome \& Yánez,2018:38)

En tal sentido, la superficie forestal del planeta disminuye velozmente, el aire puro, el agua limpia o la tierra sin contaminar empiezan a ser bienes escasos. Disminuye la seguridad alimentaria a causa del control de las semillas, los fertilizantes y los monocultivos por parte de las grandes empresas multinacionales. Aumenta la posibilidad de contraer las enfermedades que acompañan al progreso (cáncer, estrés, soledad, alergia...) (Herrero, 2006:155)

El Código orgánico del ambiente manifiesta respecto a la disposición final de los desechos sólidos urbanos lo siguiente: El derecho de las personas a vivir en un ambiente sano y ecológicamente equilibrado, así como proteger los derechos de la naturaleza para la realización del buen vivir (Art.1) Además de promover el efectivo goce de los derechos de la naturaleza y de las personas, comunas, comunidades, pueblos, nacionalidades y colectivos a vivir en un ambiente sano y ecológicamente equilibrado. (Art.4) (Ministerio del ambiente, 2018)

Los problemas jurídicos y ambientales transcienden directamente en progreso social, económico y ambiental de una nación, por ello es necesario el desarrollo efectivo de la tutela en materia de protección ambiental, ya que la degradación ambiental vulnera derechos individuales y colectivos, lo que lleva al Estado a su intervención que permita garantizar el derecho al buen vivir. La Constitución del 2008 indica en los siguientes artículos los siguiente:

Artículo 83. Son deberes y responsabilidades de las ecuatorianas y los ecuatorianos, sin perjuicio de otros previstos en la Constitución y la ley:

1. Acatar y cumplir la Constitución, la ley y las decisiones legítimas de autoridad competente.

2. Ama killa, ama llulla, ama shwa. No ser ocioso, no mentir, no robar.

3. Defender la integridad territorial del Ecuador y sus recursos naturales.

4. Colaborar en el mantenimiento de la paz y de la seguridad.

5. Respetar los derechos humanos y luchar por su cumplimiento.

6. Respetar los derechos de la naturaleza, preservar un ambiente sano y utilizar los recursos naturales de modo racional, sustentable y sostenible. 
7. Promover el bien común y anteponer el interés general al interés particular, conforme al buen vivir.

8. Administrar honradamente y con apego irrestricto a la ley el patrimonio público, y denunciar y combatir los actos de corrupción.

9. Practicar la justicia y la solidaridad en el ejercicio de sus derechos y en el disfrute de bienes y servicios.

10. Promover la unidad y la igualdad en la diversidad y en las relaciones interculturales.

11. Asumir las funciones públicas como un servicio a la colectividad y rendir cuentas a la sociedad y a la autoridad, de acuerdo con la ley.

12. Ejercer la profesión u oficio con sujeción a la ética.

13. Conservar el patrimonio cultural y natural del país, y cuidar y mantener los bienes públicos.

14. Respetar y reconocer las diferencias étnicas, nacionales, sociales, generacionales, de género, y la orientación e identidad sexual.

15. Cooperar con el Estado y la comunidad en la seguridad social, y pagar los tributos establecidos por la ley.

16. Asistir, alimentar, educar y cuidar a las hijas e hijos. Este deber es corresponsabilidad de madres y padres en igual proporción, y corresponderá también a las hijas e hijos cuando las madres y padres lo necesiten.

17. Participar en la vida política, cívica y comunitaria del país, de manera honesta y transparente.

Del articulo trascrito utsupra, se destacan los literales $1,3,5,6,7$ y 13 que manifiestan la preocupación por la conservación y cuidado del medio ambiente. 


\section{Alexandra Dolores Molina-Manzo; Génesis Gaibor-Verdezoto; Diana Rosero-Mora}

Así mismo el artículo 277 se muestra a continuación: Para la consecución del buen vivir, serán deberes generales del Estado:

1.Garantizar los derechos de las personas, las colectividades y la naturaleza.

2.Dirigir, planificar y regular el proceso de desarrollo.

3.Generar y ejecutar las políticas públicas, y controlar y sancionar su incumplimiento. 4.Producir bienes, crear y mantener infraestructura y proveer servicios públicos.

5. Impulsar el desarrollo de las actividades económicas mediante un orden jurídico e instituciones políticas que las promuevan, fomenten y defiendan mediante el cumplimiento de la Constitución y la ley.

6.Promover e impulsar la ciencia, la tecnología, las artes, los saberes ancestrales y en general las actividades de la iniciativa creativa comunitaria, asociativa, cooperativa y privada

Otro texto jurídico de gran importancia es el Código Orgánico de Organización Territorial, Autonomía y Descentralización, que rompe los paradigmas en el manejo de la gestión ambiental, del cuidado y protección del medio ambiente, ahora otorgando competencias a los Gobiernos Autónomo Descentralizados (GADs.).

El derecho ambiental, con relación a la naturaleza, pretende delimitar con las disposiciones la preservación y conservación del medio ambiente, además, de prevenir sucesos que ocasionen daños irreversibles a la naturaleza; es por ello, que se han designado competencias a los GADs a nivel nacional para que, mediante ellas, se pueda sancionar a las personas o a las entidades e instituciones que vayan en contra de las normas que preservan el medio ambiente.

La contaminación hídrica del Cantón Montalvo de la Provincia de Los Ríos, yace por las diversas actividades realizadas por los seres humanos, las que suelen repercutir en afectaciones a los ríos, esteros y quebradas provocadas por materiales químicos, microorganismos, aguas residuales y residuos industriales. De esta manera se puede definir al medio ambiente como el cumulo de valores ambientales comprendidos en el compendio de todo aquello que nos rodea, establecidos en valores naturales, sociales y culturales. El hombre mismo es parte del medio ambiente. Esto en un sentido amplio o general. De forma particular, es todo aquello donde se desarrolla la vida de los seres vivos, así como del conglomerado que hace posible la vida en el planeta tierra. El 
medio ambiente es el lugar donde habita el hombre y, por ende, necesita las garantías necesarias para preservarlo y que se convierta en un lugar seguro para las generaciones actuales y futuras.

El objetivo de la presente investigación fue fundamentar jurídica y teóricamente, que la contaminación ambiental y la falta de ordenanzas municipales es una causa de vulneración a los derechos de la naturaleza

\section{METODOLOGÍA}

La presente investigación se desarrolló desde el paradigma cuantitativa y la perspectiva del método analítico-sintético, lo que permito trabajar a partir de un objeto de estudio (normativa ambiental), la descomposición analítica de sus partes para luego ser interpretadas. Se procedió metodológicamente desde un tipo descriptivo documental con diseño bibliográfico, se extrajo de los documentos legales escrutados las ideas centrales y fueron analizados mediante la técnica de análisis de contenido. Para complementar se procedió a la determinación de la muestra de una población de 2601 habitantes del Cantón Montalvo de la cual se obtuvo 81 personas, a las cuales se les aplico un cuestionario. Cuyos resultados fueron ordenados y presentados mediante gráficas. 
Iustitia Socialis. Revista Arbitrada de Ciencias Jurídicas.

Año V. Vol. V. N³. Edición Especial. 2020-III:

Universidad Regional Autónoma de los Andes

Hecho el depósito de Ley: FA2016000064

ISSN: 2542-3371

FUNDACIÓN KOINONIA (F.K). Santa Ana de Coro, Venezuela

Alexandra Dolores Molina-Manzo; Génesis Gaibor-Verdezoto; Diana Rosero-Mora

\section{RESULTADOS}

A continuación, se muestran los resultados obtenidos de la encuesta aplicada,

Tabla. 1.

1.- ¿Conoce usted que es una Ordenanza Municipal?

\begin{tabular}{|c|c|c|}
\hline Opciones & Número & Porcentaje \\
\hline SI & 60 & $86 \%$ \\
\hline NO & 10 & $14 \%$ \\
\hline TOTAL & 70 & $100 \%$ \\
\hline
\end{tabular}

Elaboración Propia (2021).

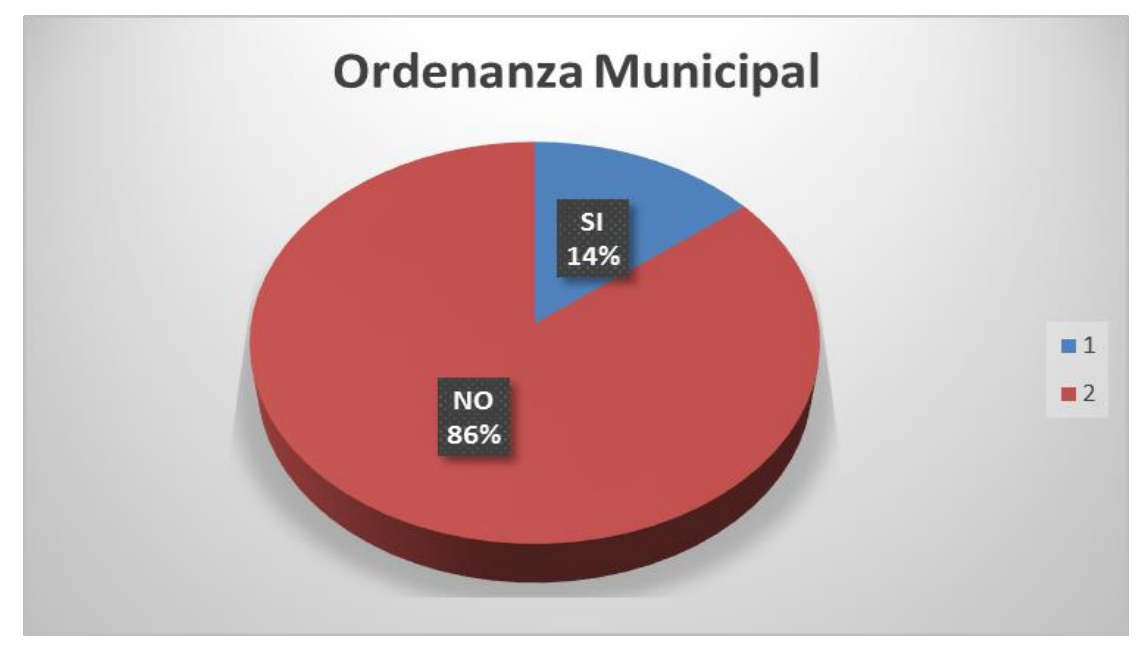

Grafica 1.

Elaboración Propia (2020). 
De la mayoría de los encuestados manifestaron que si conocen lo que es una Ordenanza Municipal.

\section{Tabla 2.}

2.- ¿Cree usted que deben existir normas ambientales?

\begin{tabular}{|c|c|c|}
\hline Opciones & Número & Porcentaje \\
\hline SI & 70 & $100 \%$ \\
\hline NO & 0 & $0 \%$ \\
\hline TOTAL & 70 & $100 \%$ \\
\hline
\end{tabular}

Elaboración Propia (2021).

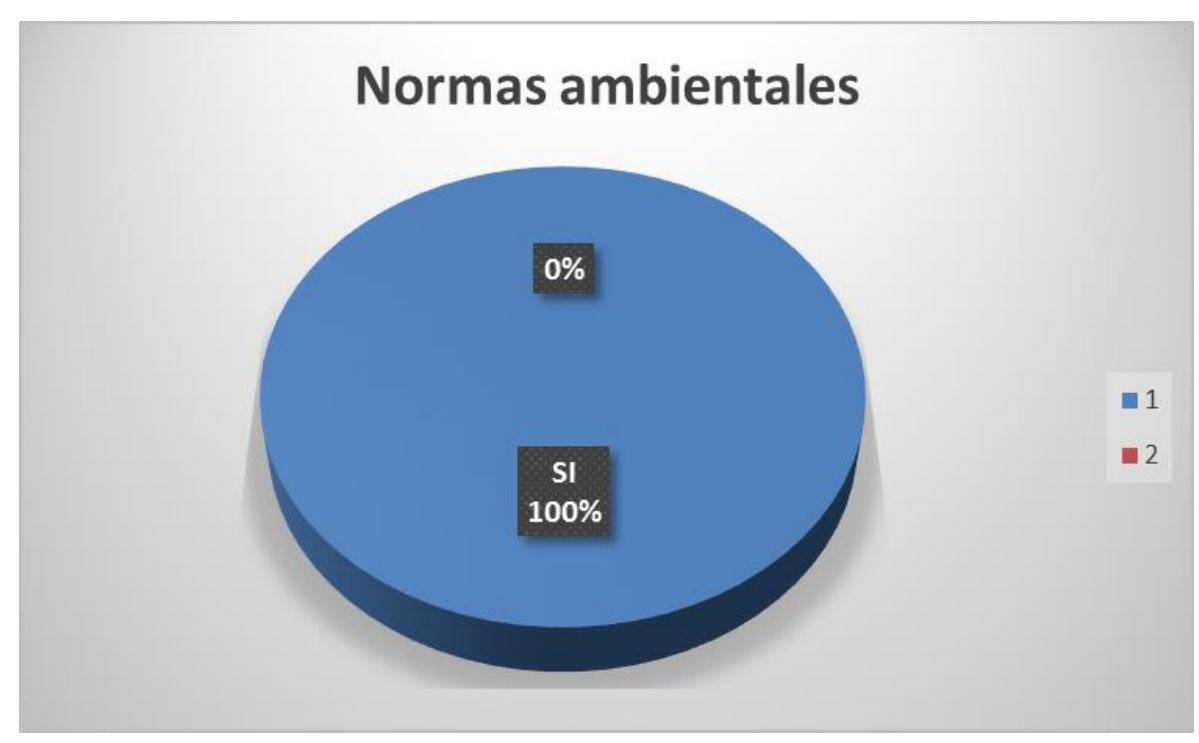

\section{Gráfica 2.}

Elaboración Propia (2021).

Con lo que se ha encuestado el $100 \%$ de los encuestados indican que, si deben existir normas ambientales, emitidas por el GAD Municipal de Montalvo. 


\section{DISCUSIÓN}

La gestión del agua en Ecuador es exclusivamente pública y es la autoridad Ambiental, la responsable de valorar y administrar los servicios ambientales considerados dentro de ellos, la purificación, filtración y desintoxicación del aire, agua y suelo, correspondiéndole a esta misma autoridad lo relativo al manejo de los desechos sólidos, asunto este reconocido de deficiente y por demás, responsable de la contaminación de aguas. El 70 \% de las ciudades no tiene sistema de manejo de desechos sólidos y arrojan las basuras a los ríos, quebradas y cuerpos de agua. (Gonzaga-Añazco, Castro -Perdomo \& López-Cavajar, 2017:38)

Por otro lado, el informe INEC-Banco Mundial Indicadores ODS de agua, saneamiento e higiene en Ecuador 2016, nos muestra lo siguiente:

Ecuador presenta altos porcentajes de cobertura de servicios de agua y saneamiento a nivel nacional. En el año 2012, la cobertura de servicios de agua potable a nivel de hogares fue del $74,5 \%$ y la cobertura de servicios de saneamiento fue del $93,2 \%$ (Senplades, 2013). Las tasas de cobertura a nivel de hogares incrementaron a diciembre de 2016, el acceso a red pública alcanzó el 83,6\%, mientras que el acceso a saneamiento se incrementó al $95,2 \%$ ese mismo año4. Cabe recalcar que, para la cuantificación de la cobertura de agua potable, solo se contabilizó el agua proveniente de conexiones municipales, excluyéndose otro tipo de fuentes por tubería, como lo provisto por juntas de agua. En el caso del indicador de saneamiento, la estadística de cobertura se refiere al porcentaje de hogares del sector rural que cuentan con sistemas adecuados 5 de eliminación de excretas. En el sector urbano, este indicador alcanza valores cercanos al $100 \%$. Las estadísticas mencionadas constituyen insumos para la política pública, donde los márgenes de intervención se definen en base a los umbrales de estos indicadores. Sin embargo, la normativa nacional referente al manejo del agua y saneamiento contemplan otras características además de la cobertura. La Constitución Política del Ecuador, el Plan Nacional para el Buen Vivir, La Ley Orgánica de Recursos Hídricos, Usos y Aprovechamiento del Agua, y otros cuerpos legales, mencionan como deber del Estado, la provisión continua y universal de servicios de agua potable y saneamiento de calidad. 
Un ejemplo a seguir es la organización Amazónica que contempla y persigue proteger también el Agua y recursos hídricos, incorporando el manejo integral de las cuencas hídricas en la planificación y ordenamiento territorial y algo fundamental es la incorporación de mecanismos de monitoreo ambiental comunitario. (Pozo-Calderón, E., Jiménez-Cargua, J., Chávez-Cadena M., \& Chamba-Orellana, S.,2020:84).

La discusión sobre los requerimientos de ordenanzas por parte de los miembros de los GADs, es una tarea pendiente y que debe llamar a la reflexión por la importancia que reviste, así como las consecuencias negativas que implica la vulneración de los derechos fundamentales de los ciudadanos.

\section{CONCLUSIONES}

En la investigación se encontró que el Gobierno Autónomo Descentralizado Municipal del Cantón Montalvo no cuenta con ordenanzas que ayuden a disminuir, proteger y sancionar a los contaminantes de ríos, esteros y quebradas y peor aún con trabajos que garanticen un medio ambiente adecuado para los pobladores del Cantón.

Luego de tener una reunión con el Alcalde y su equipo de trabajo se llegó a la conclusión unánime de que el Cantón Montalvo necesita de carácter urgente una Ordenanza que sancione a sus contaminantes y proteja los derechos hídricos del Cantón que a su vez contribuye un atractivo turístico e ingreso económico para la ciudadanía. No obstante, la contaminación hídrica aumenta paulatinamente, no solo por los contaminantes, sino también por el desconocimiento y la falta de conciencia de los ciudadanos al momento de utilizar las fuentes hídricas del Cantón.

\section{FINANCIAMIENTO}

No monetario.

\section{AGRADECIMIENTO}

A la Universidad Regional Autónoma de los Andes; por motivar el desarrollo de la Investigación. 


\section{REFERENCIAS CONSULTADAS}

Asamblea Nacional Constituyente de la República del Ecuador, (2008). Constitución de la República del Ecuador. Montecristi. Registro Oficial 449 de 20-oct-2008. Recuperado de https://n9.cl/sia

Asamblea Nacional Constituyente. (2010). Código orgánico de organización territorial Autonomía y descentralización. [Organic code of territorial organization Autonomy and decentralization]. Registro Oficial Suplemento 303 de 19-oct2010 Recuperado de: https://n9.cl/d9vg

Asamblea Constituyente. (2017). Código Orgánico del Ambiente. [Organic Environment Code]. Quito, Pichincha, Ecuador: Registro Oficial 983.

Gonzaga-Añazco, S., Castro-Perdomo, N., \& López-Calvajar, G. (2017). El abasto de agua potable y la salud comunitaria: Machala, Ecuador. Estudio de caso [Drinking water supply and community health: Machala, Ecuador. Case study] Revista Universidad y Sociedad, 9(1), 218-223. Recuperado de: https://n9.cl/w37kd

Herrero, Y. (2006). El movimiento ecologista ante el deterioro global: retos y utopías. [The environmental movement in the face of global deterioration: challenges and utopias]. Psychosocial Intervention, 15(2), 149-166.

Instituto Nacional de Estadísticas y Censo (2016). Estudios temáticos Indicadores ODS de agua, saneamiento e higiene en Ecuador. [Thematic studies SDP indicators of water, sanitation and hygiene in Ecuador]

Ministerio del Ambiente (2018). Dirección Nacional de Control Ambiental. https://n9.cl/8xw76

Ministerio del Ambiente (2017). Texto Unificado de Legislación Secundaria de Medio Ambiente. [Unified Text of Secondary Environmental Legislation]. Decreto Ejecutivo 3516 Registro Oficial Edición Especial 2 de 31-mar-2003

Pozo-Calderón, E., Jiménez-Cargua, J., Chávez-Cadena M., \& Chamba-Orellana, S. (2020) Legislación Ambiental, Regionalización y Presupuesto Ley Orgánica de Planificación Integral de Circunscripción Territorial Especial Amazónica. [Environmental Legislation, Regionalization and Budget Organic Law of Integral Planning of Amazonas Special Territorial Circumscription]. IUSTITIA SOCIALIS.5(8),73-90. http://dx.doi.org/10.35381/racji.v5i8.562 
Iustitia Socialis. Revista Arbitrada de Ciencias Jurídicas.

Año V. Vol. V. N³. Edición Especial. 2020-III

Universidad Regional Autónoma de los Andes

Hecho el depósito de Ley: FA2016000064

ISSN: 2542-3371

FUNDACIÓN KOINONIA (F.K). Santa Ana de Coro, Venezuela

Alexandra Dolores Molina-Manzo; Génesis Gaibor-Verdezoto; Diana Rosero-Mora

Terneus-Jácome, E. \& Yánez, P. (2018). Principios fundamentales en torno a la calidad del agua, el uso de bioindicadores acuáticos y la restauración ecológica fluvial en ecuador. [Fundamental principles around water quality, the use of aquatic bioindicators and river ecological restoration at equator]. LA GRANJA: Revista de Ciencias de la Vida. 27(1),36-50. http://doi.org/10.17163/lgr.n27.2018.03

(C2020 por los autores. Este artículo es de acceso abierto y distribuido según los términos y condiciones de la licencia Creative Commons Atribución-NoComercial-Compartirlgual 4.0 Internacional (CC BY-NC-SA 4.0) (https://creativecommons.org/licenses/by-nc-sa/4.0/). 